\title{
An outbreak of norovirus GI-6 infection following a wedding in North West England
}

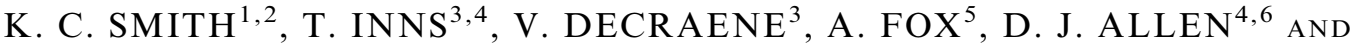 \\ A. $\mathrm{SHAH}^{1 *}$ \\ ${ }^{1}$ Health Protection Team, Public Health England, North West, UK \\ ${ }^{2}$ School of Public Health, Health Education North West, Liverpool, UK \\ ${ }^{3}$ Field Epidemiology Service, National Infections Service, Public Health England, Liverpool, UK \\ ${ }^{4}$ NIHR Health Protection Research Unit in Gastrointestinal Infections, UK \\ ${ }^{5}$ Food Water and Environmental Microbiology Laboratory, Public Health England, Preston, UK \\ ${ }^{6}$ Virus Reference Department, National Infections Service, Public Health England, Colindale, London, UK
}

Received 23 July 2016; Final revision 3 November 2016; Accepted 8 December 2016;

first published online 30 January 2017

\section{SUMMARY}

In August 2015 a gastroenteritis outbreak occurred following a wedding. An outbreak investigation was undertaken and a cohort study was conducted using an online survey. Of 140 guests, 134 received the survey and 113 responded (84.3\% response rate). Seventy respondents met the case definition of vomiting and/or diarrhoea within $72 \mathrm{~h}$ of the wedding $(61.9 \%$ attack rate). Fifteen exposures were associated with illness; on stratification, all were confounded by the ham hock starter. Multivariable analysis showed a significant association with exposure to ham hock (risk ratio 6.62, 95\% confidence interval 2.19-20.03). Eight guests and two catering staff submitted stool samples. All tested positive for norovirus GI-6 infection, including a food handler who had vomiting less than $48 \mathrm{~h}$ before the wedding. A single genotype was detected among all samples, suggesting a single source of contamination. The transmission pattern suggested pointsource exposure. The most plausible cause of the outbreak was transmission from an infected food handler via contaminated food. This highlights the importance of appropriate exclusions for symptomatic food handlers. Additionally, the food handler's stool sample was submitted 7 days after symptom resolution. The potential for extended viral excretion, and the extremely low infective dose of norovirus, may mean that current exclusion guidelines are not of sufficient duration.

Key words: Caliciviruses, gastrointestinal infections, Norwalk agent and related viruses, outbreaks.

\section{INTRODUCTION}

Norovirus is a highly infectious viral pathogen of the Caliciviridae family that is a common cause of gastroenteritis in adults and children [1]. Norovirus was

\footnotetext{
* Author for correspondence: Dr A. Shah, Health Protection Team, Public Health England, North West, UK.

(Email: Anjila.Shah@phe.gov.uk)
}

found to be the most common cause of infectious intestinal disease in the UK during the IID2 longitudinal study [2]. Additionally, using 2008-2009 prices, norovirus was estimated to have a total annual cost to patients and the health service of $£ 81$ million (95\% confidence interval £63-106 million), which, based on the same study, was a larger economic burden than that of Campylobacter and rotavirus combined [3]. 
Infected individuals excrete the virus in faeces and vomit, and continue to do so for several days, before, during and after their symptoms. Asymptomatic infection also occurs, and these individuals can also shed the virus, posing a particular challenge to infection control [4-6]. Humans are the only known reservoir for human norovirus infections, but contamination of food, water and the environment is possible, so indirect transmission of norovirus also occurs [4, 7-9].

This paper describes an investigation into a food-associated norovirus outbreak linked to a wedding in North West England. In August 2015, Public Health England (PHE) were contacted about a wedding, with reports that over 50 of the 140 guests were experiencing symptoms of illness including diarrhoea, vomiting and headache. An outbreak was declared and an Outbreak Control Team (OCT) was convened to undertake an outbreak investigation, identify the source of the outbreak and ensure appropriate control measures were implemented to prevent further transmission.

\section{METHODS}

\section{Epidemiological investigations}

Cases were defined as any person who attended the wedding and experienced vomiting and/or diarrhoea with an onset date in the $72 \mathrm{~h}$ following the wedding.

A retrospective cohort study was carried out to support the outbreak investigation, using a list of food and drink items provided by the caterers. A web-based survey was sent to people who attended the wedding, and was first emailed to the bride's family for circulation 6 days after the wedding. Respondents could complete the survey on behalf of other guests, such as young children, and were asked to indicate if they had done so. Staff who worked at the wedding were excluded from the cohort as their exposures were believed to be systematically different from guests.

Demographics and exposure among cases and noncases were described and compared. Univariable and multivariable analysis was undertaken. Poisson regression with robust error variance was used to quantify associations between exposures and illness [risk ratios (RRs) with 95\% confidence intervals (CIs)].

For each item with a $P$ value $<0.05$ on univariable analysis, univariable regression was conducted to explore if any of the associations showed a dose-response relationship. For canapés, respondents were asked to select from $0,1-2$ or $\geqslant 3$. For other food items, respondents were asked to select from none, a taste or a portion. Exposure to drink items and the chocolate truffles was not quantified in the survey questions.

The multivariable analysis was undertaken using forward stepwise modelling. Only those items which significantly improved model parsimony, as measured using Akaike's Information Criterion, were included in the final model.

All analyses were undertaken using Stata v. 12.1 (StataCorp., USA).

\section{Microbiological investigations}

Stool specimens were submitted by two catering staff and eight wedding guests. The eight wedding guests came from four different households. The 10 samples were collected between 5 and 13 days after the wedding. All 10 samples were tested for bacteria and enteric viruses. Environmental swabs were also taken at the caterer's premises and the wedding venue, and were tested for hygiene indicator organisms (including Enterobacteriaceae, E. coli and coagulase-positive Staphylococci) and norovirus.

For faecal specimens, total nucleic acid was obtained using QIAxtractor automated nucleic acid extraction platform (Qiagen, USA). For environmental specimens, total nucleic acid was extracted using a GTC-silica method performed as described previously [10]. From total nucleic acid, RNA was converted to cDNA in a random-primed reverse transcription reaction and norovirus detected using real-time PCR methods as described previously [11].

Noroviruses were genotyped through nucleic acid sequence analysis of a region encoding the S-domain of the capsid (region C) [12, 13]. Sequence analysis was performed using Bionumerics v. 6.1 (Applied Maths, Belgium). Sequences from this study were submitted to GenBank under accession numbers KY056133-KY056140.

\section{Environmental investigations}

Environmental Health Officers (EHOs) conducted environmental inspections of the caterer's premises and the wedding venue, along with review of the caterer's Food Safety Management System and discussions with staff from both companies. No food samples were submitted as none were remaining from the event. 


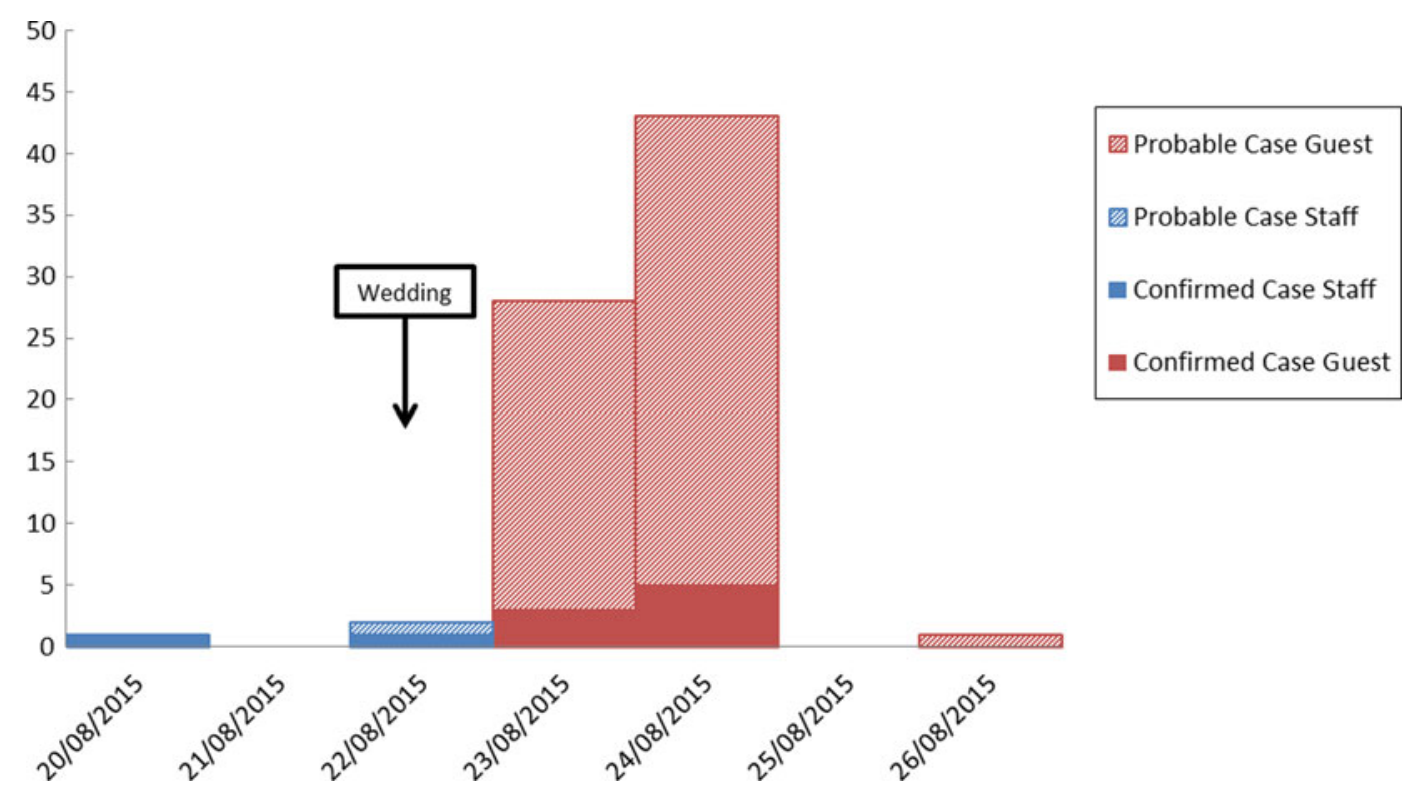

Fig. 1. Epidemic curve showing onset date of illness in persons associated with the wedding, August 2015.

\section{RESULTS}

\section{Epidemiological Investigations}

The survey was sent to 134 of 140 wedding guests. In total, $113(83.4 \%)$ individuals responded to the survey, of whom $60(53 \cdot 1 \%)$ were male. Age was reported by 89 respondents, with an average age of 39 (range 4-88) years.

Of the survey respondents, $76(67 \cdot 3 \%)$ reported illness (Fig. 1). However, one gave an onset date $>72$ $\mathrm{h}$ after the event and five reported illness but did not have symptoms of vomiting or diarrhoea, so were not included as cases. Therefore, of the 113 respondents, $70(61.9 \%)$ met the case definition.

Of the 70 cases, $50(71.4 \%)$ experienced vomiting, and $64(91 \cdot 4 \%)$ experienced diarrhoea. Nausea was reported by $65(92.9 \%)$ and abdominal pain was reported by $51(72 \cdot 9 \%)$ people. Over half $(54 \cdot 3 \%)$ of cases also experienced fever, and half $(50 \cdot 0 \%)$ reported headache, with a small number $(7 \cdot 1 \%)$ reporting bloody diarrhoea. The most commonly reported symptom duration was 2 days $(48 \cdot 6 \%)$, with duration ranging from $<1$ day to $\geqslant 5$ days.

There were no significant differences between the cases and non-cases by age or gender. Individuals who attended the event during the whole day, which included the canape reception and wedding breakfast as well as the evening buffet, had an estimated RR of 4.42 (95\% CI 1.49-11.99) of becoming a case compared to those who attended only the evening buffet.
The analytical study identified 15 items where there was strong evidence of association between exposure and being a case, as shown in Table 1. At a univariable level, the highest RRs were for the ham hock starter (RR 7.63, 95\% CI 2.60-22.32), the meat sausages served to adults (RR 6.91, 95\% CI 2.37-20.15), and the mashed potato (RR $5 \cdot 35,95 \%$ CI $2 \cdot 15-13 \cdot 31$ ) served as an accompaniment to all of the main courses. Of the 70 cases, $67(95.7 \%)$ ate the ham hock and meat sausages and $65(92.8 \%)$ ate the mashed potatoes.

For the dose-response analysis the canapés either showed a lower effect at a higher dose, or did not have strong evidence for a dose-response relationship. The ham hock starter, meat sausages, and leeks all showed strong dose-response relationships. The mashed potato, peas and lemon meringue all showed some dose-response. The bread basket showed no evidence of a dose-response relationship.

The mashed potato, peas, leeks, bread basket, lemon meringue, chocolate truffles and all of the canapés were confounded by the ham hock. The champagne was also confounded by the ham hock with some evidence of effect modification. The mineral water showed possible confounding.

Due to strong collinearity between the ham hock and the meat sausages it was not possible to assess confounding. Consequently, for subsequent modelling, the ham hock was used and the meat sausages were excluded in view of the higher univariable risk associated with the ham hock. 
Table 1. Univariable analysis

\begin{tabular}{|c|c|c|c|c|c|c|c|c|}
\hline \multirow[b]{2}{*}{ Exposure } & \multicolumn{3}{|c|}{ Exposed } & \multicolumn{3}{|c|}{ Unexposed } & \multirow[b]{2}{*}{ RR $(95 \%$ CI) } & \multirow[b]{2}{*}{$P$} \\
\hline & Total & Case & $\mathrm{AR} \%$ & Total & Case & $\mathrm{AR} \%$ & & \\
\hline Ham hock & 82 & 67 & $81 \cdot 7$ & 28 & 3 & $10 \cdot 7$ & $7 \cdot 63(2 \cdot 60-22 \cdot 32)$ & $<0.0001$ \\
\hline Meat sausages & 84 & 67 & $79 \cdot 8$ & 26 & 3 & $11 \cdot 5$ & $6 \cdot 91(2 \cdot 37-20 \cdot 15)$ & $<0.0001$ \\
\hline Mashed potato & 82 & 65 & $79 \cdot 3$ & 27 & 4 & $14 \cdot 8$ & $5 \cdot 35(2 \cdot 15-13 \cdot 31)$ & $<0.0001$ \\
\hline Lemon meringue & 80 & 63 & $78 \cdot 8$ & 31 & 7 & $22 \cdot 6$ & $3 \cdot 49(1 \cdot 80-6 \cdot 76)$ & $<0.0001$ \\
\hline Peas & 69 & 56 & $81 \cdot 2$ & 38 & 11 & $29 \cdot 0$ & $2 \cdot 80(1 \cdot 68-4 \cdot 67)$ & $<0.0001$ \\
\hline Leeks & 59 & 49 & $83 \cdot 1$ & 44 & 15 & $34 \cdot 1$ & $2 \cdot 44(1 \cdot 59-3 \cdot 73)$ & $<0.0001$ \\
\hline Chicken skewers & 59 & 48 & $81 \cdot 4$ & 47 & 19 & $40 \cdot 4$ & $2 \cdot 01(1 \cdot 39-2 \cdot 91)$ & $<0.0001$ \\
\hline Champagne & 67 & 53 & $79 \cdot 1$ & 40 & 16 & $40 \cdot 0$ & $1.98(1.33-2.95)$ & $<0.0001$ \\
\hline Bread basket & 66 & 51 & $77 \cdot 3$ & 41 & 16 & $39 \cdot 0$ & $1.98(1.32-2.97)$ & $<0.0001$ \\
\hline Cheese risotto balls & 57 & 45 & $79 \cdot 0$ & 47 & 19 & $40 \cdot 4$ & $1 \cdot 95(1 \cdot 35-2 \cdot 83)$ & $<0.0001$ \\
\hline Tandoori chicken & 52 & 42 & $80 \cdot 7$ & 55 & 25 & $55 \cdot 5$ & $1 \cdot 78(1 \cdot 29-2 \cdot 44)$ & $<0.0001$ \\
\hline Spiced lamb & 54 & 41 & $75 \cdot 9$ & 54 & 27 & $50 \cdot 0$ & $1 \cdot 52(1 \cdot 12-2 \cdot 06)$ & $0 \cdot 009$ \\
\hline Mushroom risotto & 42 & 33 & $78 \cdot 6$ & 63 & 33 & $52 \cdot 4$ & $1 \cdot 50(1 \cdot 13-1 \cdot 99)$ & $0 \cdot 008$ \\
\hline Mineral water & 39 & 29 & $74 \cdot 4$ & 60 & 30 & $50 \cdot 0$ & $1.49(1 \cdot 09-2 \cdot 03)$ & $0 \cdot 021$ \\
\hline Chocolate truffles & 42 & 32 & $76 \cdot 2$ & 68 & 38 & $55 \cdot 9$ & $1 \cdot 36(1 \cdot 04-1 \cdot 79)$ & $0 \cdot 041$ \\
\hline
\end{tabular}

$\mathrm{AR}$, Attack rate; RR, risk ratio; $\mathrm{CI}$, confidence interval.

The final multivariable model is shown in Table 2; inclusion of the leeks, the chicken skewers, the cheese risotto, and the mineral water significantly improved the model. When adjusted for these items, there was still a strongly significant relationship between becoming a case and having been exposed to the ham hock (adjusted RR 6.62, 95\% CI 2.19-20.03).

In addition to the quantitative analysis, qualitative comments were also received via the survey. Of 45 text responses, ten people $(22 \cdot 2 \%)$ mentioned the ham hock, with comments including concerns about the serving temperature, the smell, the taste, the texture and the meat being undercooked. One person $(2 \cdot 2 \%)$ commented that the lemon meringue was sour, but no other comments were received about specific food items.

\section{Microbiological investigations}

\section{Stool samples}

The eight specimens submitted from wedding guests for testing were negative for any pathogenic bacteria; however, genogroup I norovirus was detected in all eight samples.

Two staff members who catered the event submitted specimens and genogroup I norovirus was detected in both. One of these individuals reported symptoms prior to the event, and the other reported symptoms that began on the day of the event. A third symptomatic staff member did not submit a specimen.
Table 2. Multivariable model

\begin{tabular}{llr}
\hline \hline Exposure & Adjusted RR $(95 \% \mathrm{CI})$ & \multicolumn{1}{c}{$P$} \\
\hline Ham hock & $6 \cdot 62(2 \cdot 19-20 \cdot 03)$ & $<0 \cdot 001$ \\
Leeks & $1 \cdot 15(0 \cdot 84-1 \cdot 57)$ & $0 \cdot 397$ \\
Chicken skewers & $0.91(0.69-1 \cdot 20)$ & 0.912 \\
Cheese risotto balls & $1 \cdot 01(0 \cdot 77-1 \cdot 33)$ & 0.943 \\
Mineral water & $1 \cdot 27(0 \cdot 98-1 \cdot 64)$ & $0 \cdot 071$ \\
\hline \hline
\end{tabular}

$\mathrm{RR}$, Risk ratio; CI, confidence interval.

\section{Environmental samples}

Of the 17 swabs taken from the caterers' premises, seven were tested for hygiene indicator organisms and 10 were tested for norovirus. A hygiene indicator sample from the chiller handle tested positive for Enterobacteriaceae.

Twenty swabs were taken from the venue and tested for norovirus. A genogroup II norovirus was detected in one swab from the ladies' toilet and one from the chiller handle. The venue had not been open to the public between the wedding and the sampling. However, venue staff had access to the chiller, which they used to store milk, and the public toilets in the intervening period.

\section{Additional testing}

All of the positive samples were sent to the national reference laboratory for further typing. Further analysis was conducted for all 10 stool samples (eight 


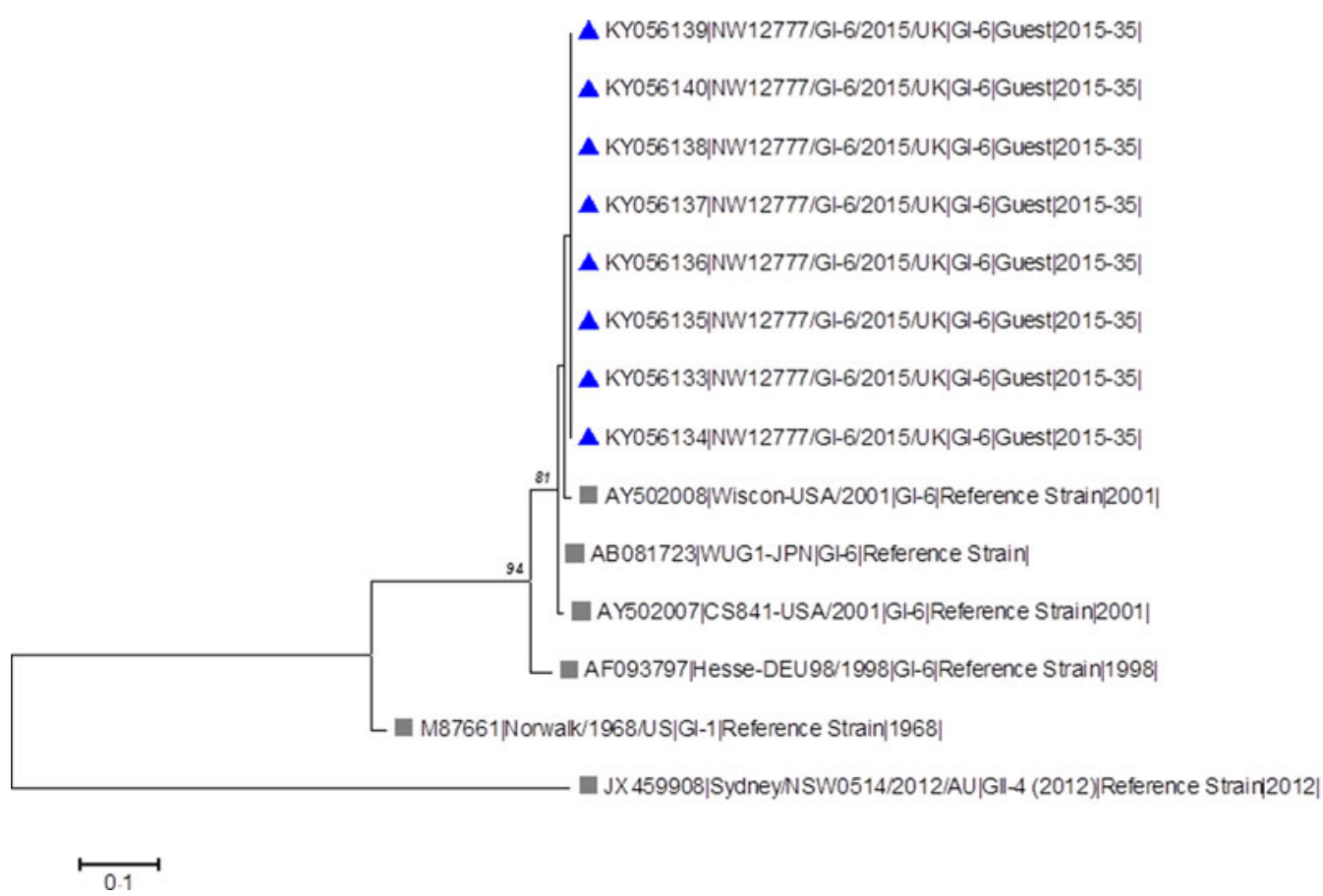

Fig. 2. Phylogenetic tree derived from eight norovirus partial capsid (ORF2) sequences from eight specimens (accession numbers: KY056133-KY056140) collected from symptomatic guests. The tree was constructed using the maximum likelihood method in MEGA6 [20]. Branch length represents number of substitutions per site. Specimens from cases are marked with blue triangles; reference sequences obtained from GenBank are marked with grey squares. Numbers on branches show bootstrap values $>80 \%$ (1000 replicates).

guests and two staff members). The genogroup I virus was characterized as genotype 6 (GI-6); all sequences were identical (Fig. 2). No further analysis was possible on the environmental swabs.

\section{Environmental investigations}

Wedding venue

An EHO visit did not identify any areas of concern. The venue staff were not involved with catering and none reported being unwell before, during or after the wedding. The norovirus identified on environmental swabs was different to that involved in the outbreak; however, in view of its presence the EHO provided reminders of handwashing advice to venue staff.

\section{Caterer}

The investigation identified a number of areas of concern. The chef who provided catering on the wedding day had vomiting which had resolved $<48 \mathrm{~h}$ earlier, and a GI-6 norovirus was detected in a faecal specimen submitted 5 days after the wedding. Two staff were ill at the wedding and were quarantined at the venue until the end of the event; one submitted a specimen in which GI-6 norovirus was detected.

The ham hock starter was cooked 2 days prior to the wedding by a chef who did not attend the event and did not report illness. It was transported to the venue in moulds on the wedding day, when it was plated and garnished, without further cooking, by the chef who subsequently tested positive for norovirus. There were no records of control checks to assess storage, transport, hot holding or service temperatures. A hand wash basin at the caterer's premises was next to a raw meat preparation area.

As a result of the findings of the outbreak investigation, a warning letter was issued by the local authority environmental health team.

\section{DISCUSSION}

Our investigation concluded that this outbreak of norovirus gastroenteritis was associated with a ham hock dish that had been prepared by a food handler who had vomiting $<48 \mathrm{~h}$ before the wedding. A GI- 6 norovirus was detected in the faeces of all cases and food handlers tested. The food handler who had vomiting 
$<48 \mathrm{~h}$ before the wedding submitted a specimen 5 days after the wedding in which a GI-6 virus was detected, having had no further symptoms since the vomiting resolved.

The epidemiological analysis indicated a strong association between the ham hock and becoming a case. The collinearity between the ham hock and the meat sausages meant that the sausages were excluded from further analysis, and this is a limitation of the study. It was also noted that three of the cases did not report consuming the ham hock. The lack of any food samples for testing also meant that it was not possible to prove that any items were contaminated with norovirus. However, the epidemiological analysis, including the qualitative feedback in the survey, alongside the findings of the environmental investigation, lead to the conclusion that the ham hock was the most likely vehicle of transmission. Furthermore, the positive stool sample in a food handler who was symptomatic prior to the wedding provided a plausible source of contamination of the food.

Although GI-6 norovirus strains are detected in the UK each year, over the last 10 years they have only accounted for $1 \cdot 0-1 \cdot 5 \%$ of all norovirus strains identified (D. J. Allen, personal communication). A paper on diversity of norovirus in the North of England included two outbreaks which were designated as GI-6 [13]. Publications have also reported outbreaks linked with GI-6 in Hesse, Germany [14], the USA [15] and Australia [16].

The Centers for Disease Control and Prevention in the USA, reported in 2013 that there had been an increase in the proportion of GI-6 outbreaks in recent years, with $7 \cdot 7 \%$ of 2012 outbreaks being due to GI-6 compared to $1.4 \%$ in 2010 . Additionally, it was found that GI-6 outbreaks were more likely to be associated with food, and had summer seasonality, compared to non-GI-6 outbreaks [17]. This correlates with the timing and mode of transmission of the outbreak investigations detailed in this paper.

The IID2 study has identified that the burden of norovirus in the UK is likely to be much higher than that identified by national surveillance. It is estimated that for every norovirus case identified through surveillance, there were 12.7 GP consultations $(95 \%$ CI $8 \cdot 8-18 \cdot 3)$, and 288 cases in the community $(95 \%$ CI 239-346) [2]. Furthermore, as GI-6 outbreaks have been shown to be less likely to occur in healthcare settings than non-GI-6 outbreaks [17], it may be that there is an element of underreporting compared to the strains that commonly occur in healthcare settings, due to a decreased likelihood of symptom reporting and sample submission.

The food-associated transmission in this outbreak was associated with contamination by an infected food handler, who was not appropriately excluded for $48 \mathrm{~h}$ following the resolution of symptoms. There have been multiple papers outlining the role of postsymptomatic food handlers in the transmission of norovirus and subsequent outbreaks [6-8]. This outbreak further highlights the importance of appropriate exclusions for symptomatic food handlers.

While the food handler returned to work $<48 \mathrm{~h}$ after symptom resolution, the positive stool sample 7 days after reported resolution of symptoms also reinforces the potential for extended viral excretion in those infected. It has been demonstrated that postsymptomatic viral shedding of norovirus may last longer than the typical 48-h exclusion period $[18,19]$. Particularly in view of the low infective dose of norovirus, this extended, post-symptomatic excretion, could well be an ongoing risk for transmission. Therefore, as previously highlighted, in food handlers with gastroenteritis symptoms, there should be further consideration of whether the existing exclusion guidelines are sufficient [5].

\section{CONCLUSIONS}

This study found that this norovirus outbreak was food-associated transmission of norovirus (GI-6), associated with inappropriate exclusion of an infected food handler. The summer seasonality and mode of transmission are in keeping with broader trends related to GI-6 in the USA. The extended postsymptomatic viral excretion in the food handler highlights the need for further consideration of appropriate duration of exclusion in this group.

\section{ACKNOWLEDGEMENTS}

The authors thank the OCT, particularly the EHOs and laboratory staff, for their significant contribution to the outbreak investigation.

The research was funded by the National Institute for Health Research Health Protection Research Unit (NIHR HPRU) in Gastrointestinal Infections at University of Liverpool in partnership with Public Health England (PHE), in collaboration with University of East Anglia, University of Oxford and the Institute of Food Research. D.J.A. is based at PHE. The views expressed are those of the author(s) 
and not necessarily those of the NHS, the NIHR, the Department of Health or PHE.

This research received no specific grant from any funding agency, commercial or not-for-profit sectors.

\section{DECLARATION OF INTEREST}

None.

\section{REFERENCES}

1. Norovirus Working Group. Guidance for the management of norovirus infection in cruise ships. London: Health Protection Agency, 2007.

2. Tam CC, et al. Longitudinal study of infectious intestinal disease in the UK (IID2 study): incidence in the community and presenting to general practice. Gut 2012; 61: 69-77.

3. Tam CC, O'Brien SJ. Economic cost of campylobacter, norovirus and rotavirus disease in the United Kingdom. PLoS ONE. Published online: 1 February 2016. doi:10.1371/journal.pone.0138526.

4. Hawker J, et al. Communicable Disease Control and Health Protection Handbook, 3rd edn. Chichester: Wiley-Blackwell, 2012.

5. Barrabeig I, et al. Foodborne norovirus outbreak: the role of an asymptomatic food handler. $B M C$ Infectious Diseases. Published online: 15 September 2010.doi:10.1186/1471-2334-10-269.

6. Franck KT, et al. Sources of calicivirus contamination in foodborne outbreaks in Denmark, 2005-2011 - the role of the asymptomatic food handler. Journal of Infectious Diseases 2015; 211: 563-570.

7. Patterson T, Hutchings P, Palmer S. Outbreak of SRSV gastroenteritis at an international conference traced to food handled by a post-symptomatic caterer. Epidemiology and Infection 1993; 111: 157-162.

8. Thornley CN, et al. Multiple outbreaks of a novel norovirus GII.4 linked to an infected post-symptomatic food handler. Epidemiology and Infection 2013; 141: 1585-1597.

9. Einöder-Moreno M, et al. Non-heat-treated frozen raspberries the most likely vehicle of a norovirus outbreak in Oslo, Norway, November 2013. Epidemiology and Infection. Published online: 16 February 2016. doi:10.1017/S0950268816000194.

10. Boom R, et al. Rapid and simple method for purification of nucleic acids. Journal of Clinical Microbiology 1990; 28: 495-503.

11. Kageyama T, et al. Broadly reactive and highly sensitive assay for Norwalk-like viruses based on real-time quantitative reverse transcription-PCR. Journal of Clinical Microbiology 2003; 41: 1458-1557.

12. Kojima $\mathbf{S}$, et al. Genogroup-specific PCR primers for detection of Norwalk-like viruses. Journal of Virological Methods 2002; 100: 107-114.

13. Gallimore CI, et al. Diversity of noroviruses cocirculating in the North of England from 1998 to 2001. Journal of Clinical Microbiology 2004; 42: 1396-1401.

14. Maunula L, Miettinen IT, von Bonsdorff CH. Norovirus outbreaks from drinking water. Emerging Infectious Diseases 2005; 11: 1716-1721.

15. Xerry J, et al. Genetic characterization of genogroup I norovirus in outbreaks of gastroenteritis. Journal of Clinical Microbiology 2010; 48: 2560-2562.

16. Bruggink LD, Dunbar NL, Marshall JA. Norovirus genotype diversity in community-based sporadic gastroenteritis incidents: a five-year study. Journal of Medical Virology 2015; 87: 961-969.

17. Leshem E, et al. Genotype GI.6 norovirus, United States, 2010-2012. Emerging Infectious Diseases 2013; 19: $1317-1320$.

18. Rockx B, et al. Natural history of human calicivirus infection: a prospective cohort study. Clinical Infectious Diseases 2002; 35: 246-253.

19. Atmar RL, et al. Norwalk virus shedding after experimental human infection. Emerging Infectious Diseases 2008; 15: 1553-1557.

20. Tamura K, et al. MEGA6: Molecular Evolutionary Genetics Analysis version 6.0. Molecular Biology and Evolution 2013; 30: 2725-2729. 